\title{
Post-diagnosis diet and the risk of progression
}

T he marked discrepancy in survival between men with localized prostate cancer and those with advanced disease makes the search for modifiable factors that influence progression of prime importance. Two new studies implicate ingestion of eggs, poultry with skin, and certain fatty acids in advancement of prostatic malignancy.

Speculation about interactions between diet and cancer began several decades ago. Interest in this area was initially piqued by epidemiological observations of marked variation in disease incidence between populations with contrasting eating habits. Several associations have been detected between prostate cancer occurrence and nutritive factors, such as saturated fat, soy proteins, and lycopenes. The bulk of research effort has, however, been directed towards risk of disease development, rather than likelihood of progression.

In 2006, June Chan and colleagues from the University of California, San Francisco, reported that prostate cancer was less likely to progress if patients increased their consumption of fish and tomato sauce after diagnosis. These authors hypothesized that red meat would have the opposite effect. To their surprise, their new data have disproved this theory.

Published online in the American Journal of Clinical Nutrition, the results of Chan et al's prospective analysis of nearly 1,300 men enrolled in the Diet and Lifestyle substudy of CaPSURE implicate consumption of eggs and of poultry with skin-but not red meat-in progression and recurrence of localized or regional prostate cancer. The risk of disease-related death, bone metastases, biochemical recurrence, or initiation of secondary treatment (127 events in total) more than doubled among men who ate the most eggs or poultry with skin.

The authors speculate that cooked poultry skin contains high levels of heterocyclic amines, mutagens that induce prostatic carcinomas in rats. Elevated plasma concentrations of choline might explain the

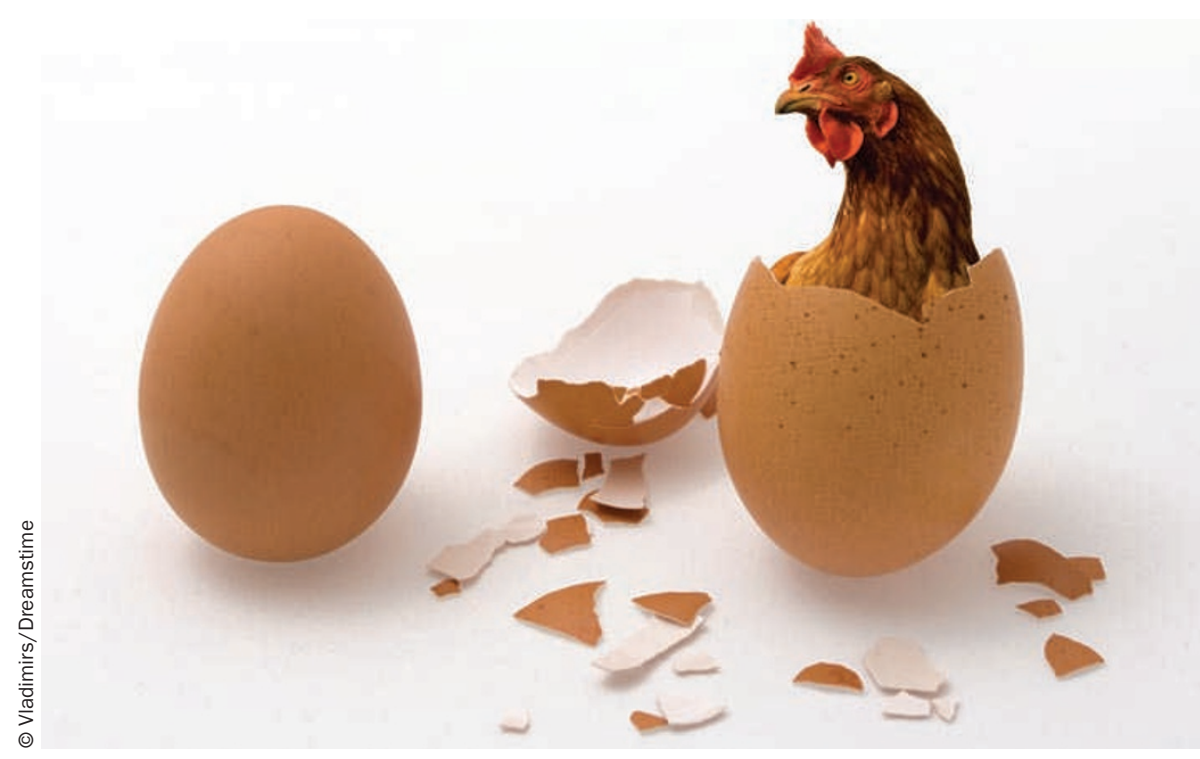

association between eggs and the increased likelihood of disease progression.

"Our results are entirely novel", lead author Erin Richman told Medscape Oncology. While stopping short of recommending that clinicians advise patients to avoid these foods, Richman pointed out "that cancer survivors are at increased risk of heart disease and diabetes, and limiting consumption of poultry with skin is consistent with current guidelines for reducing heart disease risk".

The impressively rapid impact on prostate-cancer-cell growth of lowering dietary fat intake has been elegantly demonstrated by William Aronson and colleagues. The team randomized 20 overweight men with treatment-naive prostate cancer to either a typical Western diet, or to a low-fat, high-fiber regimen supplemented with soy protein. All meals were prepared by study staff. In vitro proliferation of LNCaP cells dropped by $16 \%$ following incubation with serum collected from patients on the low-fat diet.

Lead author Aronson finds it "quite remarkable that in [just] 4 weeks, a dietary intervention can change the growth factors in human serum that stimulate prostate cancer growth". Correlation analysis showed decreased levels of omega- 6 and increased levels of omega-3 unsaturated fatty acids to be associated with the diet-induced slowing of prostate-cancer-cell growth. It has been proposed that cancer cells lack the enzymes required to metabolize unsaturated fatty acids. Conversion of the unmetabolized acids into reactive oxygen species could destroy tumor cells.

Aronson et al's ex vivo proliferation bioassay is a fast and novel means of assessing the impact of dietary interventions on prostate cancer progression. It could prove to be a useful surrogate biomarker for clinically meaningful end points in future long-term studies of modifiable lifestyle factors, which are notoriously expensive and labor-intensive. Indeed, these investigators are currently trialing a low-fat diet supplemented with fish oil in men who undergo radical prostatectomy. Publication of results later this year is eagerly awaited.

Suzanne J. Farley

\footnotetext{
Original articles Richman, E. L. et al. Intakes of meat, fish, poultry, and eggs and risk of prostate cancer progression. Am. J. Clin. Nutr. doi:10.3945/ajcn.2009.28474 | Aronson, W. J. et al. Growth inhibitory effect of low fat diet on prostate cancer cells: results of a prospective, randomized dietary intervention trial in men with prostate cancer. J. Urol. 183, 345-350 (2010)
} 